\title{
On Approximation of Entropy Solutions for One System of Nonlinear Hyperbolic Conservation Laws with Impulse Source Terms
}

\author{
Ciro D’Apice, ${ }^{1}$ Peter I. Kogut, ${ }^{2}$ and Rosanna Manzo' \\ ${ }^{1}$ Dipartimento di Ingegneria Elettronica e Ingegneria Informatica, Università degli Studi di Salerno, \\ Via Ponte don Melillo, 84084 Fisciano, Italy \\ ${ }^{2}$ Department of Differential Equations, Dnipropetrovsk National University, Gagarin Avenue, 72, \\ 49010 Dnipropetrovsk, Ukraine
}

Correspondence should be addressed to Rosanna Manzo, manzo@diima.unisa.it

Received 9 September 2010; Accepted 18 December 2010

Academic Editor: Yoshito Ohta

Copyright (C) 2010 Ciro D’Apice et al. This is an open access article distributed under the Creative Commons Attribution License, which permits unrestricted use, distribution, and reproduction in any medium, provided the original work is properly cited.

\begin{abstract}
We study one class of nonlinear fluid dynamic models with impulse source terms. The model consists of a system of two hyperbolic conservation laws: a nonlinear conservation law for the goods density and a linear evolution equation for the processing rate. We consider the case when influx-rates in the second equation take the form of impulse functions. Using the vanishing viscosity method and the so-called principle of fictitious controls, we show that entropy solutions to the original Cauchy problem can be approximated by optimal solutions of special optimization problems.
\end{abstract}

\section{Introduction}

The main goal of this paper is to approximate entropy solutions to a Cauchy problem for the system of nonlinear balance laws with an impulse source term. Conservation laws, taking the form of hyperbolic partial differential equations, appear in a variety of applications that offer control or identification of parameters, including the control of traffic and water flows, the modeling of supply chains, gas pipelines, blood flows, and so forth. The analysis of conservation laws is a very active research area. The main difficulty in dealing with them is the fact that the solution of such systems may develop discontinuities (after a finite time), that propagate in time even for smooth initial and boundary conditions (see [1-3]). Usually such solutions can be formed by the so-called rarefaction or shock waves. Therefore, it makes a sense to consider a more flexible notion of solutions, which are physically meaningful and whose admissibility issue is related to the notions of entropy and energy.
We analyze the following initial value problem for the system of nonlinear conservation laws

$$
\begin{gathered}
\rho_{t}+(f(\mu, \rho))_{x}=0, \quad(t, x) \in \Omega_{T}=(0, T) \times \mathbb{R}, \\
\mu_{t}-\mu_{x}=u(t, x), \quad(t, x) \in \Omega_{T}, \\
\rho(0, x)=\rho_{0}(x), \quad \mu(0, x)=\mu_{0}(x), \quad x \in \mathbb{R} .
\end{gathered}
$$

Throughout this paper we suppose that the structure of the source term $u(t, x)$ is prescribed, namely,

$$
\begin{aligned}
& u=u(t, x)=\sum_{i=1}^{N} u_{i}(t) \delta_{\tau_{i}}(x), \\
& \text { with }-\infty<a<\tau_{1}<\cdots<\tau_{N}<b<+\infty,
\end{aligned}
$$

where the functions $\left\{u_{i} \in L^{2}(0, T)\right\}_{i=1}^{N}$ can play the role of control factors, and $\delta_{\tau_{i}}$ denote the Dirac measures located at the points $\tau_{i}$.

In the recent applications of the model (1) to the supply chain problem [4], $\rho=\rho(t, x)$ represents the density of 
objects or the concentration of a physical quantity processed by the supply chain (modeled by a real line $\mathbb{R}$ ), and $\mu=$ $\mu(t, x)$ is the processing rate. However, to the best knowledge of authors, the existence and uniqueness of entropy solutions to the problems of conservation laws with impulse controls is an open problem even for the simplest situation. Thus our prime interest is to discuss the approximation approach to the construction of entropy solutions for the above problem. To this end, we apply the vanishing viscosity method and the so-called principle of fictitious controls. We prove that entropy solutions to the Cauchy problem (1)-(2) can be approximated by optimal solutions of special optimization problems. Namely, we introduce the following penalized optimization problem

$$
\begin{aligned}
I_{\mathcal{E}}\left(v^{\mathcal{\varepsilon}}, \rho^{\varepsilon}\right)= & \left\|v^{\varepsilon}\right\|_{L^{2}(0, T ; \mathcal{M}(\mathcal{O}))}^{2} \\
& +\mathcal{\varepsilon}^{-1}\left\|\left(f_{2}\left(\mu^{\varepsilon}\right)\right)_{x}-v^{\varepsilon}\right\|_{L^{2}\left(0, T ; H^{-1}(\mathcal{O})\right)} \longrightarrow \inf
\end{aligned}
$$

subject to the constraints

$$
\begin{gathered}
\rho_{t}^{\varepsilon}(t, x)-\varepsilon \rho_{x x}^{\varepsilon}(t, x)+\left(f_{1}\left(\rho^{\varepsilon}(t, x)\right)\right)_{x}=v(t, x), \quad(t, x) \in \Omega_{T}, \\
\mu_{t}^{\varepsilon}(t, x)-\varepsilon \mu_{x x}^{\varepsilon}(t, x)-\mu_{x}^{\varepsilon}(t, x)=u(t, x), \quad(t, x) \in \Omega_{T}, \\
\rho^{\varepsilon}(0, x)=\rho_{0}(x), \quad \mu^{\varepsilon}(0, x)=\mu_{0}(x), \quad x \in \mathbb{R}, \\
u(t, x)=u_{1}(t) \delta_{\tau_{1}}(x)+u_{2}(t) \delta_{\tau_{2}}(x)+\cdots+u_{N}(t) \delta_{\tau_{N}}(x), \\
u_{i} \in L^{2}(0, T), \quad \forall i=\{1, \ldots, N\}, \\
v \in L^{2}(0, T ; \mathcal{M}(\mathbb{R})), \\
\rho^{\varepsilon}(t, x)=0, \quad \mu^{\varepsilon}(t, x)=0, \quad \text { on }(0, T) \times \partial \mathcal{O},
\end{gathered}
$$

where $v=v(t, x)$ is a fictitious control. We carry out the analysis of this problem and show that under some additional assumptions every cluster pair $\left(v^{*}, \rho^{*}\right.$ ) (in an appropriate topology) of the sequence $\left\{\left(v_{0}^{\varepsilon}, \rho_{0}^{\varepsilon}\right) \in \Xi_{\varepsilon}\right\}_{\varepsilon>0}$ of optimal solutions to the penalized problem (3)-(4) is an entropy solution $\left(u^{*}, \rho^{*}, \mu^{*}\right)$ to the Cauchy problem (1).

\section{Notation and Preliminaries}

Let $a$ and $b$ be two fixed constants such that $-\infty<a<b<$ $+\infty$. For a given $T>0$ we set $\Omega_{T}=(0, T) \times \mathbb{R}$ and $\Omega=$ $(0, T) \times(a, b)$. Let $L_{\mathrm{loc}}^{p}\left(\Omega_{T}\right)$, with $1 \leq p \leq \infty$, be the locally convex space of all measurable functions $g: \Omega_{T} \rightarrow \mathbb{R}$ such that $\left.g\right|_{(0, T) \times K} \in L^{p}((0, T) \times K)$ for all compact sets $K \subset \mathbb{R}$.

Let $\mathcal{M}(\mathbb{R})$ be the set of all Radon measures on $\mathbb{R}$, that is, $\mu \in \mathcal{M}(\mathbb{R})$ if $\mu$ is a countably additive set function defined on the Borel subsets of $\mathbb{R}$ such that $\mu$ is finite on every compact subset of $\mathbb{R}$. We say that a sequence of Radon measures $\left\{\mu_{k}\right\}_{k \in \mathbb{N}}$ converges weakly- $*$ to a measure $\mu \in \mathcal{M}(\mathbb{R})$ (in symbols $\mu_{k} \stackrel{*}{\rightarrow} \mu$ ) if

$$
\lim _{k \rightarrow \infty} \int_{\mathbb{R}} \varphi d \mu_{k}=\int_{\mathbb{R}} \varphi d \mu, \quad \forall \varphi \in C_{0}(\mathbb{R}) .
$$

A subset $\mathfrak{M}$ of $\mathcal{M}(\mathbb{R})$ is called to be bounded if for every compact set $K \subset \mathbb{R}$ we have

$$
\sup _{\mu \in \mathfrak{M}}|\mu|(K)<+\infty
$$

where $|\mu|$ denotes the total variation of $\mu$. The following compactness result for measures is well known.

Proposition 1. Let $\left\{\mu_{k}\right\}_{k \in \mathbb{N}}$ be a bounded sequence of Radon measures on $\mathbb{R}$. Then there exist a subsequence $\left\{\mu_{k_{j}}\right\}_{j \in \mathbb{N}}$ and a Radon measure $\mu \in \mathcal{M}(\mathbb{R})$ such that $\mu_{k_{j}} \stackrel{*}{\rightarrow} \mu$.

According to the Riesz theory, every Radon measure $\mu$ on $\mathbb{R}$ can be identified with an element of the dual space $\left(C_{0}(\mathbb{R})\right)^{\prime}$, that is, $\mu$ is a linear form on $C_{0}(\mathbb{R})$ and for every compact set $K \subset \mathbb{R}$ there exists a constant $C>0$ depending only on $K$ and $\mu$ such that

$$
|\langle\mu, f\rangle| \leq C\|f\|_{C(K)}, \quad \forall f \in C_{0}(\mathbb{R}) \text { with supp } f \subseteq K .
$$

As an example of a Radon measure on $\mathbb{R}$, we consider the following one. Let $\left\{a_{k}\right\}_{k \in \mathbb{N}}$ and $\left\{b_{k}\right\}_{k \in \mathbb{N}}$ be two sequences in $\mathbb{R}$ such that $\sum_{k=1}^{\infty}\left|a_{k}\right| \leq \mathbf{C}<+\infty$. Let $\delta_{c}$ be the Dirac measure located at the point $c \in \mathbb{R}$, that is, this measure is defined as follows

$$
\left\langle\delta_{c}, \varphi\right\rangle=\int_{\mathbb{R}} \delta_{c}(x) \varphi(x) d x:=\varphi(c), \quad \forall \varphi \in C_{0}(\mathbb{R}) .
$$

Since

$$
\left|\sum_{k=1}^{\infty} a_{k} \varphi\left(b_{k}\right)\right| \leq\left(\sum_{k=1}^{\infty}\left|a_{k}\right|\right)\|\varphi\|_{C(\mathbb{R})} \leq \mathbf{C}\|\varphi\|_{C(\mathbb{R})}
$$

for every continuous function with compact support $\varphi \in$ $C_{0}(\mathbb{R})$, it follows that the linear form

$$
\mu^{*}=\sum_{k=1}^{\infty} a_{k} \delta_{b_{k}}
$$

is continuous on $C_{0}(\mathbb{R})$. Hence $\mu^{*}$ is an element of the space of Radon measures $\mathcal{M}(\mathbb{R})$.

Let $\mathcal{O}$ be a bounded open subset of $\mathbb{R}$. Let $f: \mathcal{O} \rightarrow \mathbb{R}$ be an element of $L^{1}(\mathcal{O})$. Define

$$
\begin{aligned}
& \int_{\mathcal{O}}|D f| \\
& =\sup \left\{\int_{\mathcal{O}} f \varphi^{\prime} d x: \varphi \in C_{0}^{1}(\mathcal{O}),|\varphi(x)| \leq 1 \text { for } x \in \mathcal{O}\right\} .
\end{aligned}
$$

According to the Radon-Nikodym theorem, if $\int_{\mathcal{O}}|D f|<$ $+\infty$ then the distribution $D f$ is a measure and there exist a function $f^{\prime} \in L^{1}(\mathcal{O})$ and a measure $D_{s} f$, singular with respect to the one-dimensional Lebesgue measure $\mathcal{L}\lfloor\mathcal{O}$ restricted to $\mathcal{O}$, such that

$$
D f=f^{\prime} \mathcal{L}\left\lfloor\mathcal{O}+D_{s} f .\right.
$$


Definition 2. A function $f \in L^{1}(\mathcal{O})$ is said to have a bounded variation in $\mathcal{O}$ if the derivative $D f$ exists in the sense of distributions and belongs to the class of Radon measures with bounded total variation, that is, $\int_{\mathcal{O}}|D f|<+\infty$. By $\operatorname{BV}(\mathcal{O})$ we denote the space of all functions in $L^{1}(\mathcal{O})$ with bounded variation.

Under the norm

$$
\|f\|_{\mathrm{BV}(\mathcal{O})}=\|f\|_{L^{1}(\mathcal{O})}+\int_{\mathcal{O}}|D f|
$$

$\mathrm{BV}(\mathcal{O})$ is a Banach space. The following compactness result for BV-functions is well known.

Proposition 3. The uniformly bounded sets in BV-norm are relatively compact in $L^{1}(\mathcal{O})$, that is, if $\left\{f_{k}\right\}_{k=1}^{\infty} \subset B V(\mathcal{O})$ and $\sup _{k \in \mathbb{N}}\left\|f_{k}\right\|_{B V(\mathcal{O})}<+\infty$, then there exists a subsequence of $\left\{f_{k}\right\}_{k=1}^{\infty}$ strongly converging in $L^{1}(\mathcal{O})$ to some $f \in B V(\mathcal{O})$.

Definition 4. A sequence $\left\{f_{k}\right\}_{k=1}^{\infty} \subset \mathrm{BV}(\mathcal{O})$ weakly converges to some $f \in \operatorname{BV}(\mathcal{O})$, and we write $f_{k} \rightarrow f$ if and only if the two following conditions hold: $f_{k} \rightarrow f$ strongly in $L^{1}(\mathcal{O})$, and $D f_{k} \rightarrow D f$ weakly* in $\mathcal{M}(\mathcal{O})$.

In the following proposition we give a compactness result related to this convergence, together with the lower semicontinuity property (see [5]).

Proposition 5. Let $\left\{f_{k}\right\}_{k=1}^{\infty}$ be a sequence in $B V(\mathcal{O})$ strongly converging to some $f$ in $L^{1}(\mathcal{O})$ and satisfying $\sup _{k \in \mathbb{N}} \int_{\mathcal{O}}\left|D f_{k}\right|+\infty$. Then

(i) $f \in B V(\mathcal{O})$ and $\int_{\mathcal{O}}|D f| \leq \liminf _{k \rightarrow \infty} \int_{\mathcal{O}}\left|D f_{k}\right|$;

(ii) $f_{k} \rightarrow f$ in $B V(\mathcal{O})$.

\section{Statement of Problem and Main Motivation}

Let $\left\{\tau_{k}\right\}_{k=1}^{N} \subset \mathbb{R}$ be a given finite family of points such that $a<\tau_{1}<\cdots<\tau_{N}<b$. We focus on the following fluid dynamic model, expressed by the nonlinear inhomogeneous hyperbolic conservation laws:

$$
\begin{gathered}
\rho_{t}+(f(\mu, \rho))_{x}=0, \quad(t, x) \in \Omega_{T}, \\
\mu_{t}-\mu_{x}=u(t, x), \quad(t, x) \in \Omega_{T}, \\
\rho(0, x)=\rho_{0}(x), \quad \mu(0, x)=\mu_{0}(x), \quad x \in \mathbb{R},
\end{gathered}
$$

where the source term is subjected to the following constraints:

$$
\begin{gathered}
u(t, x)=u_{1}(t) \delta_{\tau_{1}}(x)+u_{2}(t) \delta_{\tau_{2}}(x)+\cdots+u_{N}(t) \delta_{\tau_{N}}(x), \\
u_{i} \in L^{2}(0, T), \quad \forall i=\{1, \ldots, N\} .
\end{gathered}
$$

Here $u_{i} \in L^{2}(0, T)$ are some external distributed sources located at the corresponding points $\tau_{i} \in(a, b), \rho_{0}, \mu_{0} \in$ $\mathrm{BV}(\mathbb{R}) \cap L^{\infty}(\mathbb{R})$ are data functions, and $f=f(\mu, \rho)=$ $f_{1}(\rho)+f_{2}(\mu)$ is a flux function.
We note that a particular case of the initial value problem (14)-(16) is a perturbed model for the supply chain (represented by a real line), where $\rho=\rho(t, x)$ represents the density of objects or the concentration of a physical quantity processed by the supply chain (modeled by a real line $\mathbb{R}$ ), $\mu=\mu(t, x)$ is the processing rate, and $u=u(t, x)$ is a source term associated with an influx-rate.

In order to give a precise description of the set of admissible source terms to the Cauchy problem (14)-(18), we note that for any function $u(t, x)$ of type (17), we have

$$
\begin{aligned}
\int_{0}^{T} \| & u(t, \cdot) \|_{\mathcal{M}(\mathbb{R})}^{2} d t \\
& =\int_{0}^{T}\left(\sup _{\|\varphi\|_{C(\mathbb{R})=1}}\langle u(t, \cdot), \varphi(\cdot)\rangle_{\mathcal{M}(\mathbb{R}), C_{0}(\mathbb{R})}\right)^{2} d t \\
& =\int_{0}^{T}\left(\sup _{\substack{\|\varphi\|_{C(\mathbb{R})}(R) \\
\varphi \in C_{0}(R)}} \sum_{k=1}^{N} u_{k}(t) \int_{\mathbb{R}} \delta_{\tau_{k}}(x) \varphi(x) d x\right)^{2} d t \\
& \leq \int_{0}^{T}\left(\sum_{k=1}^{N} u_{k}(t)\right)^{2} d t \\
& \leq N \sum_{k=1}^{N}\left\|u_{k}\right\|_{L^{2}(0, T)}^{2} .
\end{aligned}
$$

Hence, it is natural to define the following class:

$$
\begin{aligned}
& \mathcal{U}_{\mathrm{ad}}=\left\{u \in L^{2}(0, T ; \mathcal{M}(\mathbb{R})) \mid u=u(t, x)\right. \\
&\text { satisfy } \left.(25)-(26) \text { for some } a<\tau_{1}<\ldots<\tau_{N}<b\right\} .
\end{aligned}
$$

Definition 6. Let $u \in \mathcal{U}_{\text {ad }}$ be a fixed source term. We say that a vector value function $Y=\left[\begin{array}{l}\rho \\ \mu\end{array}\right] \in\left[L^{2}\left(0, T ; L_{\mathrm{loc}}^{2}(\mathbb{R})\right)\right]^{2}$ is a weak solution to (14)-(16) if the identities

$$
\begin{aligned}
& \int_{0}^{T} \int_{\mathbb{R}}\left(Y \odot \frac{\partial \varphi}{\partial t}+F(Y) \odot \frac{\partial \varphi}{\partial x}\right) d x d t \\
& \quad+\int_{0}^{T} \sum_{k=1}^{N} U_{k}(t) \odot \varphi\left(\tau_{k}\right) d t=0, \\
& \lim _{t \rightarrow 0+} \frac{1}{t} \int_{0}^{t}\left|\int_{\mathbb{R}} Y(t, x) \odot \psi(x) d x-\int_{\mathbb{R}} Y_{0} \odot \psi(x) d x\right|_{\mathbb{R}^{2}} d t=0
\end{aligned}
$$

hold true for all $C_{0}^{\infty}$-functions $\varphi:[0, T] \times \mathbb{R} \rightarrow \mathbb{R}^{2}$ and $\psi: \mathbb{R} \rightarrow \mathbb{R}^{2}$ with compact supports in $(0, T) \times \mathbb{R}$ and $\mathbb{R}$, respectively. Here

$$
Y_{0}=\left[\begin{array}{l}
\rho_{0} \\
\mu_{0}
\end{array}\right], \quad U_{k}(t)=\left[\begin{array}{c}
0 \\
u_{k}(t)
\end{array}\right], \quad F(Y)=\left[\begin{array}{c}
f(\mu, \rho) \\
-\mu
\end{array}\right],
$$

and the symbol $\odot$ denotes the tensor product $\left[\begin{array}{l}a_{1} \\ a_{2}\end{array}\right] \odot\left[\begin{array}{l}b_{1} \\ b_{2}\end{array}\right]=$ $\left[\begin{array}{l}a_{1} b_{1} \\ a_{2} b_{2}\end{array}\right]$. 
The characteristic feature of the initial value problem (14)-(16) is that even for arbitrary smooth functions $\rho_{0}$, $\mu_{0}$, and smooth external sources $u_{k}, k=1, \ldots, N$, a weak solution $Y(t, x)=(\rho(t, x), \mu(t, x))$ to (14)-(16), is, in general, not unique (see $[2,3])$. Hence, in order to select the "physically" relevant solution, some additional conditions must be imposed. Following [2, 3, 6], we can introduce the entropy-admissibility condition, coming from physical considerations.

Definition 7. A $C^{1}$-function $\eta: \mathbb{R}^{2} \rightarrow \mathbb{R}$ is an entropy for the system (14)-(15), if it is convex and there exists a $C^{1}$-function $q: \mathbb{R}^{2} \rightarrow \mathbb{R}$ such that

$$
D \eta(v) \cdot D F(v)=D q(v), \quad \forall v \in \mathbb{R}^{2} .
$$

The function $q: \mathbb{R}^{2} \rightarrow \mathbb{R}$ is said an entropy flux for $\eta$. The pair $(\eta, q)$ is said an entropy flux pair for the system (14)-(15).

Remark 8. Note that the $C^{1}$-functions $\eta, q$ in Definition 7 form a special family of convex entropy pairs. However, any convex function $\eta$ defined on an open set is locally Lipschitz, and therefore $D \eta$ is defined almost everywhere. This allows us to call a $C^{0}$-function $\eta$ an entropy, if there exists a sequence of $C^{1}$-entropies $\left\{\eta^{\nu}: \mathbb{R}^{2} \rightarrow \mathbb{R}\right\}_{\nu=1}^{\infty}$ converging to $\eta$ locally uniformly as $v \rightarrow \infty$. Moreover a $C^{0}$-function $q$ is a corresponding entropy flux, if there exists a sequence $\left\{q^{\nu}\right\}_{\nu=1}^{\infty}$ of $C^{1}$-entropy fluxes of $\eta^{\nu}$ converging to $q$ locally uniformly.

As a result, an entropy solution of (14)-(16) for a given $u \in U_{\text {ad }}$ can be defined as follows.

Definition 9. Let $u \in \mathcal{U}_{\mathrm{ad}}$ be a given source term with prescribed location $a<\tau_{1}<\cdots<\tau_{N}<b$. A weak solution $Y=\left[\begin{array}{l}\rho \\ \mu\end{array}\right]:[0, T] \times \mathbb{R} \rightarrow \mathbb{R}^{2}$ to the Cauchy problem (14)(16) is said entropy admissible if for any constants $k, l \in \mathbb{R}$ the entropy inequalities

$$
\begin{gathered}
\int_{0}^{T} \int_{\mathbb{R}}\left(\nu_{l}(\rho) \psi_{t}+g_{l}(\rho) \psi_{x}\right) d x d t \\
\quad-\int_{0}^{T} \int_{\mathbb{R}} \operatorname{sign}(\rho-l)\left(f_{2}(\mu)\right)_{x} \psi d x d t \geq 0, \\
\int_{0}^{T} \int_{\mathbb{R}}\left(\nu_{k}(\mu) \varphi_{t}-q_{k}(\mu) \varphi_{x}\right) d x d t \\
\quad+\sum_{i=1}^{T} \int_{0}^{T} \operatorname{sign}\left(\mu\left(t, \tau_{i}\right)-k\right) u_{i}(t) \varphi\left(t, \tau_{i}\right) d t \geq 0
\end{gathered}
$$

hold true for all positive functions $\varphi, \psi \in C_{0}^{\infty}\left(\Omega_{T}\right)$, provided that

$$
\begin{gathered}
v_{k}(\mu):=|\mu-k|, \quad q_{k}(\mu):=(\mu-k) \operatorname{sign}(\mu-k), \\
g_{l}(\rho):=\left(f_{1}(\rho)-f_{1}(l)\right) \operatorname{sign}(\rho-l) .
\end{gathered}
$$

Remark 10. Note that the existence and differential properties of entropy solutions to the Cauchy problem (14)-(16) with impulse influx-rate (17) in the sense of Definition 9 are unknown in general. To the best knowledge of authors, the problems (14)-(17) with measure data in the right hand side are not covered by the classical theory of nonlinear hyperbolic conservation laws. Moreover, we cannot assert that entropy admissible solutions $(\rho(u), \mu(u))$ to the above problem are elements of the class

$$
\left[C\left([0, T] ; L^{1}(a, b)\right) \cap L^{\infty}(\Omega) \cap L^{\infty}(0, T ; \operatorname{BV}(a, b))\right]^{2}
$$

which is a natural functional space for the scalar hyperbolic conservation laws (see $[2,7,8]$ ). Usually these properties essentially depend not only on the flux function $f(\mu, \rho)$, but also on the properties of the admissible source terms $u(t, x)$, which typically, in contrast to our case, are supposed to be bounded in $L^{\infty}\left(\Omega_{T}\right)$ and closed in $L^{1}\left(\Omega_{T}\right)$ (see $[8]$ ).

Taking this motivation into account, it is reasonable to introduce the following concept.

Definition 11. Let $u \in U_{\text {ad }}$ be a given source term. We say that a vector value function $Y=\left[\begin{array}{l}\rho \\ \mu\end{array}\right] \in\left[L^{2}\left(0, T ; L_{\text {loc }}^{2}(\mathbb{R})\right)\right]$ is an approximately entropy solution to the Cauchy problem (14)-(16) in a domain $(0, T) \times \mathcal{O}$, if $Y=\left[\begin{array}{l}\rho \\ \mu\end{array}\right]:[0, T] \times \mathbb{R} \rightarrow$ $\mathbb{R}^{2}$ is a weak solution in the sense of Definition 6 and there exists a sequence $\left\{Y^{\varepsilon}=\left[\begin{array}{l}\rho^{\varepsilon} \\ \mu^{\varepsilon}\end{array}\right]\right\}_{\varepsilon>0} \subset\left[L^{2}\left(0, T ; L^{2}(\mathcal{O})\right)\right]^{2}$ such that

(B1) $\rho^{\varepsilon} \rightarrow \rho$ and $\mu^{\varepsilon} \rightarrow \mu$ in $L^{2}\left(0, T ; L^{2}(\mathcal{O})\right)$ as $\varepsilon \rightarrow 0$;

(B2) for any constants $k, l \in \mathbb{R}$ and for all positive concave functions $\varphi \in C_{0}^{\infty}((0, T) \times \mathcal{O})$ the entropy inequalities

$$
\begin{aligned}
\int_{0}^{T} \int_{\mathcal{O}}\left(\nu_{l}\left(\rho^{\varepsilon}\right) \varphi_{t}\right. & \left.+g_{l}\left(\rho^{\varepsilon}\right) \varphi_{x}\right) d x d t \\
+\int_{0}^{T}\langle & \operatorname{sign}\left(\rho^{\varepsilon}(t, \cdot)-l\right) \\
& \times\left(f_{2}\left(\mu^{\varepsilon}(t, \cdot)\right)_{x}, \varphi(t, \cdot)\right\rangle_{\mathcal{M}(\mathcal{O}), C_{0}(\mathcal{O})} d t \geq 0,
\end{aligned}
$$

$$
\begin{aligned}
\int_{0}^{T} \int_{\mathcal{O}}( & \left.\nu_{k}\left(\mu^{\varepsilon}\right) \varphi_{t}-q_{k}\left(\mu^{\varepsilon}\right) \varphi_{x}\right) d x d t \\
& +\sum_{i=1}^{N} \int_{0}^{T} \operatorname{sign}\left(\mu^{\varepsilon}\left(t, \tau_{i}\right)-k\right) u_{i}(t) \varphi\left(t, \tau_{i}\right) d t \geq 0
\end{aligned}
$$

it hold true for every $\varepsilon>0$ with $\nu_{l}(\rho):=|\rho-l|$, $g_{l}(\rho):=\left(f_{1}(\rho)-f_{1}(l)\right) \operatorname{sign}(\rho-l), v_{k}(\mu):=|\mu-k|$ and $q_{k}(\mu):=(\mu-k) \operatorname{sign}(\mu-k)$.

\section{A Perturbation Framework}

As was mentioned above the existence and uniqueness of entropy solutions for nonlinear hyperbolic conservation laws (14)-(16) with source terms (17), where $u_{i} \in L^{2}(0, T)$ for all $i=1, \ldots, N$, and with initial distributions $\rho_{0}, \mu_{0} \in \mathrm{BV}(\mathbb{R}) \cap$ $L^{\infty}(\mathbb{R})$, is not covered by the classical theory. In view of this, 
we apply in this section the scheme of "vanishing viscosity" method and the principle of fictitious controls.

To begin with, we impose the following assumptions on the flux function:

$$
f(\mu, \rho)=f_{1}(\rho)+f_{2}(\mu),
$$

(A1) the function $f_{1}: \mathbb{R} \rightarrow \mathbb{R}$ is locally Lipschitz, that is,

$$
\left|f_{1}\left(\rho_{1}\right)-f_{1}\left(\rho_{2}\right)\right| \leq L_{\rho}\left|\rho_{1}-\rho_{2}\right|, \quad \forall \rho_{1}, \rho_{2} \in\left[-M_{\rho}, M_{\rho}\right],
$$

$f_{1}(0)=0$, and $f_{2}^{\prime}: \mathbb{R} \mapsto \mathbb{R}$ is a piecewise linear mapping.

Remark 12. As was shown in recent works [4, 9-11], a flux function of the fluid dynamic model for supply chains is the following:

$$
f(\mu, \rho)=f_{1}(\rho)+f_{2}(\mu)=\left\{\begin{array}{ll}
\rho, & \text { if } \rho<\mu \\
0, & \text { if } \rho \geq \mu
\end{array}\right\}+\left\{\begin{array}{ll}
0, & \text { if } \rho<\mu \\
\mu, & \text { if } \rho \geq \mu
\end{array}\right\} .
$$

Hence, the fulfilment of Hypothesis (A1) is obvious in this case.

Let $\varepsilon$ be a small positive parameter associated with a viscosity coefficient. Then instead of the fluid dynamic system (14)-(16), we focus on the following singular perturbed system of nonlinear PDEs:

$$
\begin{gathered}
\rho_{t}^{\varepsilon}(t, x)-\varepsilon \rho_{x x}^{\varepsilon}(t, x)+\left(f_{1}\left(\rho^{\varepsilon}(t, x)\right)\right)_{x}=v(t, x), \quad(t, x) \in \Omega_{T}, \\
\mu_{t}^{\varepsilon}(t, x)-\varepsilon \mu_{x x}^{\varepsilon}(t, x)-\mu_{x}^{\varepsilon}(t, x)=u(t, x), \quad(t, x) \in \Omega_{T}, \\
\rho^{\varepsilon}(0, x)=\rho_{0}(x), \quad \mu^{\varepsilon}(0, x)=\mu_{0}(x), \quad x \in \mathbb{R}
\end{gathered}
$$

subjected to the constraints

$$
\begin{gathered}
u(t, x)=u_{1}(t) \delta_{\tau_{1}}(x)+u_{2}(t) \delta_{\tau_{2}}(x)+\cdots+u_{N}(t) \delta_{\tau_{N}}(x) \\
u_{i} \in L^{2}(0, T), \quad \forall i=\{1, \ldots, N\} \\
v \in L^{2}(0, T ; \mathcal{M}(\mathbb{R}))
\end{gathered}
$$

where $v=v(t, x)$ is a fictitious control. By $\mathcal{V}_{\text {ad }}$ we denote the set of all fictitious controls satisfying conditions (37).

Since $\rho_{0}, \mu_{0} \in L^{1}\left(\mathbb{R}^{N}\right) \cap L^{\infty}\left(\mathbb{R}^{N}\right)$, it is natural to assume that there is a compact interval $I \subset \mathbb{R}$ such that $\rho_{0}=0$ and $\mu_{0}=0$ almost everywhere in $\mathbb{R} \backslash I$. Then taking a sufficiently big open bounded interval $\mathcal{O} \subset \mathbb{R}$ including the interval $I$, we can suppose that the rate processing $\mu^{\varepsilon}$ and the density $\rho^{\varepsilon}$ vanish at the ends of $\mathcal{O}$. As a result, we can introduce the following boundary conditions into the model (32)-(37):

$$
\rho^{\varepsilon}(t, x)=0, \quad \mu^{\varepsilon}(t, x)=0, \quad \text { on }(0, T) \times \partial \mathcal{O} .
$$

Since by the initial assumptions the influx-rate $u=$ $\sum_{k=1}^{N} u_{k}(t) \delta_{k}$ and the fictitious control $v$ belong to the space of measure data $L^{2}(0, T ; \mathcal{M}(\mathcal{O}))$, we make the notion of solution for the problem (32)-(38) precise. To this end, we give the following theorem which plays an important role in the study of partial differential equations (see [12]).

Theorem 13. Let one defines the Banach spaces:

$$
\mathcal{W}=\left\{y: y \in L^{2}\left(0, T ; H_{0}^{1}(\mathcal{O})\right), \frac{\partial y}{\partial t} \in L^{2}\left(0, T ; H^{-1}(\mathcal{O})\right)\right\},
$$

$$
\mathcal{W}_{1}=\left\{y: y \in L^{2}\left(0, T ; L^{2}(\mathcal{O})\right), \frac{\partial y}{\partial t} \in L^{2}\left(0, T ; H^{-1}(\mathcal{O})\right)\right\},
$$

equipped with the norm of the graph. Then, the following properties hold true:

(1) the embeddings $\mathcal{W} \hookrightarrow L^{2}\left(0, T ; L^{2}(\mathcal{O})\right), \mathcal{W}_{1} \hookrightarrow L^{2}(0, T$; $\left.H^{-1}(\mathcal{O})\right)$ are compact;

(2) one has the embedding

$$
\mathcal{W} \hookrightarrow C\left([0, T] ; L^{2}(\mathcal{O})\right), \quad \mathcal{W}_{1} \hookrightarrow\left([0, T] ; H^{-1}(\mathcal{O})\right),
$$

where, for $\mathbf{X}=L^{2}(\mathcal{O})$ or $\mathbf{X}=H^{-1}(\mathcal{O}), C([0, T] ; \mathbf{X})$ denotes the space of measurable functions on $[0, T] \times \mathcal{O}$ such that $y(t, \cdot) \in \mathbf{X}$ for any $t \in[0, T]$ and such that the map $t \in[0, T] \mapsto y(t, \cdot) \in \mathbf{X}$ is continuous;

(3) for any $u, v \in W$

$$
\begin{aligned}
& \frac{d}{d t} \int_{\mathcal{O}} u(t, x) v(t, x) d x \\
& =\left\langle u^{\prime}(t, \cdot), v(t, \cdot)\right\rangle_{H^{-1}(\mathcal{O}), H_{0}^{1}(\mathcal{O})} \\
& \quad+\left\langle v^{\prime}(t, \cdot), u(t, \cdot)\right\rangle_{H^{-1}(\mathcal{O}), H_{0}^{1}(\mathcal{O})}
\end{aligned}
$$

(4) let $y \in L^{2}\left(0, T ; H_{0}^{1}(\mathcal{O})\right) \cap C\left([0, T] ; L^{2}(\mathcal{O})\right)$. Then the following density result holds: for any $\delta>0$ there exists $\Phi \in C^{\infty}\left([0, T] ; C_{0}^{\infty}(\mathcal{O})\right)$, such that

$$
\|y-\Phi\|_{C\left([0, T] ; L^{2}(\mathcal{O})\right)} \leq \delta, \quad\|\nabla y-\nabla \Phi\|_{L^{2}((0, T) \times \mathcal{O})} \leq \delta .
$$

Further we note that by the Friedrichs inequality, we have

$$
\begin{aligned}
\left|\int_{\mathbb{O}} y \varphi^{\prime} d x\right| & \leq c \int_{\mathbb{O}} y^{2} d x \int_{\mathbb{O}}\left|\varphi^{\prime}\right|^{2} d x \\
& \leq c_{1} \int_{\mathbb{O}}\left|y^{\prime}\right|^{2} d x \int_{\mathbb{O}}\left|\varphi^{\prime}\right|^{2} d x, \quad \forall y, \varphi \in H_{0}^{1}(\mathcal{O}) .
\end{aligned}
$$

Hence the bilinear form $\int_{\mathbb{O}} y \varphi^{\prime} d x$ is bounded on $H_{0}^{1}(\mathcal{O})$. Moreover, this form is skew-symmetric by the identity

$$
\begin{aligned}
\int_{\mathbb{O}} y \varphi^{\prime} d x & =\int_{\mathbb{O}}(y \varphi)^{\prime} d x-\int_{\mathbb{O}} y^{\prime} \varphi d x \\
& =-\int_{\mathbb{O}} y^{\prime} \varphi d x, \quad \forall y, \varphi \in C_{0}^{\infty}(\mathcal{O}),
\end{aligned}
$$


which remains valid for all $y, \varphi \in H_{0}^{1}(\mathcal{O})$ by continuity. Then, we come to the following classical result (see $[12,13]$ ).

Theorem 14. Assume that $\mu_{0} \in B V(\mathcal{O}) \cap L^{\infty}(\mathcal{O})$ and Hypothesis (A1) holds true. Then for every $\varepsilon>0$ the initialboundary value problem (32)-(38) admits a unique solution $\left(\rho^{\varepsilon}, \mu^{\varepsilon}\right) \in \mathcal{W} \times \mathcal{W}$ satisfying the integral identities:

$$
\begin{gathered}
\int_{0}^{T} \int_{\mathcal{O}}\left[\frac{\partial \rho^{\varepsilon}}{\partial t} \psi+\varepsilon \frac{\partial \rho^{\varepsilon}}{\partial x} \frac{\partial \psi}{\partial x}-f_{1}\left(\rho^{\varepsilon}\right) \frac{\partial \psi}{\partial x}\right] d x d t \\
=\int_{0}^{T}\langle v(t, \cdot), \psi(t, \cdot)\rangle_{H^{-1}(\mathcal{O}), H_{0}^{1}(\mathcal{O})} d t, \\
\forall \psi \in L^{2}\left(0, T ; H_{0}^{1}(\mathcal{O})\right), \\
\int_{0}^{T} \int_{\mathcal{O}}\left[\frac{\partial \mu^{\varepsilon}}{\partial t} \varphi+\varepsilon \frac{\partial \mu^{\varepsilon}}{\partial x} \frac{\partial \varphi}{\partial x}+\mu^{\varepsilon} \frac{\partial \varphi}{\partial x}\right] d x d t \\
=\int_{0}^{T}\langle u(t, \cdot), \varphi(t, \cdot)\rangle_{H^{-1}(\mathcal{O}), H_{0}^{1}(\mathcal{O})} d t, \\
\forall \varphi \in L^{2}\left(0, T ; H_{0}^{1}(\mathcal{O})\right),
\end{gathered}
$$

with a priori estimates

$$
\begin{gathered}
\int_{0}^{T} \int_{\mathcal{O}}\left[\left|\rho^{\varepsilon}(t, x)\right|^{2}+\varepsilon\left|\rho_{x}^{\varepsilon}(t, x)\right|^{2}+\left|\rho_{t}^{\varepsilon}(t, x)\right|^{2}\right] d x d t \\
\leq C \int_{0}^{T} \int_{\mathcal{O}}\left[\left|\hat{g}_{t}(t, x)\right|^{2}+\left|\hat{g}_{x}(t, x)\right|^{2}\right] d x d t \\
\quad+C\|v\|_{L^{2}\left(0, T ; H^{-1}(\mathcal{O})\right)}^{2} \\
\int_{0}^{T} \int_{\mathcal{O}}\left[\left|\mu^{\varepsilon}(t, x)\right|^{2}+\varepsilon\left|\mu_{x}^{\varepsilon}(t, x)\right|^{2}+\left|\mu_{t}^{\varepsilon}(t, x)\right|^{2}\right] d x d t \\
\leq C \int_{0}^{T} \int_{\mathcal{O}}\left[\left|g_{t}(t, x)\right|^{2}+\left|g_{x}(t, x)\right|^{2}\right] d x d t \\
\quad+C\|u\|_{L^{2}\left(0, T ; H^{-1}(\mathcal{O})\right)}^{2}, \\
\int_{0}^{T}\left\|\rho_{t}^{\varepsilon}(t, \cdot)\right\|_{H^{-1}(\mathcal{O})}^{2} d t \\
\leq C\|\hat{g}\|_{W^{-1,2}((0, T) \times \mathcal{O})}^{2}+C\|v\|_{L^{2}\left(0, T ; H^{-1}(\mathcal{O})\right)}^{2}, \\
\int_{0}^{T}\left\|\mu_{t}^{\varepsilon}(t, \cdot)\right\|_{H^{-1}(\mathcal{O})}^{2} d t \\
\leq C\|g\|_{W^{-1,2}((0, T) \times \mathcal{O})}^{2}+C\|u\|_{L^{2}\left(0, T ; H^{-1}(\mathcal{O})\right)}^{2},
\end{gathered}
$$

where $C>0$ is a constant independent of $\varepsilon$ and $g, \hat{g} \in$ $W^{1,2}((0, T) \times \mathcal{O})$ are such that $\left.g\right|_{\partial \mathcal{O}}=0,\left.\hat{g}\right|_{\partial \mathcal{O}}=0, \hat{g}(0, \cdot)=$ $\rho_{0}$, and $g(0, \cdot)=\mu_{0}$ in $\mathcal{O}$ (the so-called compatibility condition).

Note that in this case

$$
\rho^{\varepsilon} \in C\left([0, T] ; L^{2}(\mathcal{O})\right), \quad \mu^{\varepsilon} \in C\left([0, T] ; L^{2}(\mathcal{O})\right)
$$

by the embedding (41), and the terms in the right-hand sides of (46)-(47) are well defined, because $H_{0}^{1}(\mathcal{O}) \subset C_{0}(\overline{\mathcal{O}})$ by the classical Sobolev Embedding Theorem. Moreover, in the one-dimensional case every Radon measure $\nu \in \mathcal{M}(\mathcal{O})$ can be identified with an element of $H^{-1}(\mathcal{O})$, that is, $\mathcal{M}(\mathcal{O}) \subset$ $H^{-1}(\mathcal{O})$. As a result, the integral identity (47) with a source term

$$
u(t, x)=\sum_{k=1}^{N} u_{k}(t) \delta_{k}(x)
$$

can be rewritten as follows:

$$
\begin{aligned}
& \int_{0}^{T} \int_{\mathcal{O}} {\left[\frac{\partial \mu^{\varepsilon}}{\partial t} \varphi+\varepsilon \frac{\partial \mu^{\varepsilon}}{\partial x} \frac{\partial \varphi}{\partial x}+\mu^{\varepsilon} \frac{\partial \varphi}{\partial x}\right] d x d t } \\
& \quad=\sum_{k=1}^{N} \int_{0}^{T} u_{k}(t) \varphi\left(t, \tau_{k}\right) d t, \quad \forall \varphi \in L^{2}\left(0, T ; H_{0}^{1}(\mathcal{O})\right) .
\end{aligned}
$$

In conclusion of this section we state the following entropy property of the weak solutions to the initialboundary value problem (32)-(38).

Lemma 15. Let $u^{*}=\sum_{k=1}^{N} u_{k}^{*}(t) \delta_{\tau_{k}} \in U_{a d}$ be a given source term with prescribed location $a<\tau_{1}<\cdots<\tau_{N}<b$. Let $\left\{\left(\rho^{\varepsilon}, \mu^{\varepsilon}\right)\right\}_{\varepsilon>0}$ be a sequence of corresponding weak solutions to the initial boundary value problem (32)-(38) where the small parameter $\varepsilon>0$ varies in a strictly decreasing sequence of positive numbers converging to 0 . Let $\left\{v^{\varepsilon} \in L^{2}(0, T\right.$; $\mathcal{M}(\mathbb{R}))\}_{\varepsilon>0}$ be a bounded sequence of fictitious controls. Assume that supposition (A1) holds true. Then for every $\varepsilon>0, k, l \in \mathbb{R}$, and for all positive concave functions $\varphi \in C_{0}^{\infty}((0, T) \times \mathcal{O})$, each of the pairs $\left(\left(\rho^{\varepsilon}, \mu^{\varepsilon}\right)\right)$ satisfies the following integral inequalities:

$$
\begin{aligned}
& \int_{0}^{T} \int_{\mathcal{O}}\left(\nu_{l}\left(\rho^{\varepsilon}\right) \varphi_{t}+g_{l}\left(\rho^{\varepsilon}\right) \varphi_{x}\right) d x d t \\
& \quad+\int_{0}^{T}\left\langle\operatorname{sign}\left(\rho^{\varepsilon}(t, \cdot)-l\right) v^{\varepsilon}(t, \cdot), \varphi(t, \cdot)\right\rangle_{\mathcal{M}(\mathcal{O}), C_{0}(\mathcal{O})} d t \geq,
\end{aligned}
$$

$$
\begin{aligned}
& \int_{0}^{T} \int_{\mathcal{O}}\left(\nu_{k}\left(\mu^{\varepsilon}\right) \varphi_{t}-q_{k}\left(\mu^{\varepsilon}\right) \varphi_{x}\right) d x d t \\
& \quad+\sum_{i=1}^{N} \int_{0}^{T} \operatorname{sign}\left(\mu^{\varepsilon}\left(t, \tau_{i}\right)-k\right) u_{i}^{*}(t) \varphi\left(t, \tau_{i}\right) d t \geq 0
\end{aligned}
$$

with $\nu_{l}(\rho):=|\rho-l|, g_{l}(\rho):=\left(f_{1}(\rho)-f_{1}(l)\right) \operatorname{sign}(\rho-l)$, $\nu_{k}(\mu):=|\mu-k|$, and $q_{k}(\mu):=(\mu-k) \operatorname{sign}(\mu-k)$.

Proof. Let $E=E(\rho) \in C^{2}(\mathbb{R})$ be any convex function. We multiply (32) by $E^{\prime}(\rho)$. Then the equalities

$$
\begin{gathered}
E^{\prime}(\rho) \rho_{t}=\frac{\partial E(\rho(t, x))}{\partial t}, \\
f_{1}^{\prime}(\rho) E^{\prime}(\rho) \rho_{x}=\frac{\partial}{\partial x}\left(\int_{k}^{\rho(t, x)} f_{1}^{\prime}(\xi) E^{\prime}(\xi) d \xi\right), \\
E^{\prime}(\rho) \rho_{x x}=(E(\rho))_{x x}-E^{\prime \prime}(\rho) \rho_{x}^{2},
\end{gathered}
$$


imply the following relation

$$
\begin{aligned}
& \left(E\left(\rho^{\varepsilon}\right)\right)_{t}+\left(\int_{k}^{\rho^{\varepsilon}} f_{1}^{\prime}(\xi) E^{\prime}(\xi) d \xi\right)_{x} \\
& =\varepsilon\left(E\left(\rho^{\varepsilon}\right)\right)_{x x}-\varepsilon E^{\prime \prime}\left(\rho^{\varepsilon}\right)\left(\rho^{\varepsilon}\right)_{x}^{2}+E^{\prime}\left(\rho^{\varepsilon}\right) v^{\varepsilon}, \text { in } \mathfrak{D}^{\prime}((0, T) \times \mathcal{O}) .
\end{aligned}
$$

By the initial assumptions, for every $\varepsilon>0$ the functions $\rho^{\varepsilon} \in$ $W$ can be zero-extended to the domain $\Omega_{T}=(0, T) \times \mathbb{R}$. Now let us multiply equality (58) by a test function $\varphi \in C_{0}^{\infty}\left(\Omega_{T}\right)$ and integrate it over $\Omega_{T}$. Using the integration by parts and the fact that $\varepsilon>0$ and $E^{\prime \prime}\left(\rho^{\varepsilon}\right) \geq 0$ a.e. in $\Omega_{T}$, we transfer all derivatives to the test function $\varphi$ :

$$
\begin{aligned}
& -\int_{0}^{T} \int_{\mathbb{R}}\left[E\left(\rho^{\varepsilon}\right) \varphi_{t}+\int_{k}^{\rho^{\varepsilon}} f_{1}^{\prime}(\xi) E^{\prime}(\xi) d \xi \varphi_{x}\right] d x d t \\
& =\varepsilon \int_{0}^{T} \int_{\mathbb{R}}\left(E\left(\rho^{\varepsilon}\right)\right) \varphi_{x x} d x d t \\
& \quad-\varepsilon \int_{0}^{T} \int_{\mathbb{R}} E^{\prime \prime}\left(\rho^{\varepsilon}\right)\left(\rho^{\varepsilon}\right)_{x}^{2} \varphi d x d t+\int_{0}^{T} \int_{\mathbb{R}} E^{\prime}\left(\rho^{\varepsilon}\right) v^{\varepsilon} \varphi d x d t \\
& \leq \varepsilon \int_{0}^{T} \int_{\mathbb{R}} E\left(\rho^{\varepsilon}\right) \varphi_{x x} d x d t+\int_{0}^{T} \int_{\mathbb{R}} E^{\prime}\left(\rho^{\varepsilon}\right) v^{\varepsilon} \varphi d x d t .
\end{aligned}
$$

Since $\rho^{\varepsilon} \in L^{2}\left(0, T ; H_{0}^{1}(\mathcal{O})\right)$ for all $\varepsilon>0$ and $H_{0}^{1}(\mathcal{O}) \hookrightarrow C(\overline{\mathcal{O}})$ by the classical Sobolev Embedding Theorem, it follows that the following term is well defined:

$$
\int_{0}^{T} \int_{\mathbb{R}} E^{\prime}\left(\rho^{\varepsilon}\right) v^{\varepsilon} \varphi d x d t=\int_{0}^{T}\left\langle E^{\prime}\left(\rho^{\varepsilon}\right) v^{\varepsilon}, \varphi\right\rangle_{\mathcal{M}(\mathbb{R}), C_{0}(\mathbb{R})} d t .
$$

Further we use the well-known trick. Let $\left\{E_{m}\right\}_{m \in \mathbb{N}}$ be a sequence of $C^{2}$-functions approximating the function $\xi \mapsto$ $|\xi-k|$ uniformly on $\mathbb{R}$. Substitute $E=E_{m}(\rho)$ in the inequality (59) and pass to the limit as $m \rightarrow \infty$. Note that we can choose $E_{m}$ in such way that $E_{m}^{\prime}$ is bounded and $E_{m}^{\prime}(\xi) \rightarrow \operatorname{sign}(\xi-k)$ for all $\xi \in \mathbb{R}, \xi \neq k$. Since $\varphi_{x} x \leq 0$ in $\Omega_{T}$ and

$$
\begin{aligned}
& \int_{k}^{\rho^{\varepsilon}} f_{1}^{\prime}(\xi) E_{m}^{\prime}(\xi) d \xi \longrightarrow \int_{k}^{\rho^{\varepsilon}} f_{1}^{\prime}(\xi) \operatorname{sign}(\xi-k) d \xi \\
& =\operatorname{sign}\left(\rho^{\varepsilon}-k\right) \int_{k}^{\rho^{\varepsilon}} f_{1}^{\prime}(\xi) d \xi=\operatorname{sign}\left(\rho^{\varepsilon}-k\right)\left(f_{1}\left(\rho^{\varepsilon}\right)-f_{1}(k)\right),
\end{aligned}
$$

it immediately leads us to the entropy inequality (55) from (59). The verification of inequality (56) can be done by similar arguments.

\section{The Penalized Optimization Problem}

In this section, for every $\varepsilon>0$ and a given influx-rate $u^{\varepsilon} \in U_{\mathrm{ad}}$, we analyze the following penalized optimization problem associated with the singular perturbed initialboundary value problem (32)-(38):

$$
\begin{aligned}
I_{\mathcal{E}}\left(v^{\varepsilon}, \rho^{\varepsilon}\right)= & \left\|v^{\varepsilon}\right\|_{L^{2}(0, T ; \mathcal{M}(\mathcal{O}))}^{2} \\
& +\mathcal{\varepsilon}^{-1}\left\|\left(f_{2}\left(\mu^{\varepsilon}\right)\right)_{x}-v^{\varepsilon}\right\|_{L^{2}\left(0, T ; H^{-1}(\mathcal{O})\right)} \longrightarrow \inf
\end{aligned}
$$

subject to the constraints (33)-(39).

Definition 16. We say that a pair $\left(v^{\varepsilon}, \rho^{\varepsilon}\right)$ is admissible to the optimization problem (62)-(63) if $v^{\varepsilon} \in L^{2}(0, T ; \mathcal{M}(\mathbb{R}))$ and $\rho^{\varepsilon}=\rho^{\varepsilon}\left(v^{\varepsilon}\right) \in \mathcal{W}$ is the corresponding weak solution to the initial boundary value problem (32), (34) $)_{1}$, and $(38)_{1}$.

Let $\Xi_{\varepsilon}$ be the set of all admissible solutions to the perturbed problem (62)-(63). As follows from Theorem 13, for every $\varepsilon>0, \Xi_{\varepsilon}$ is a nonempty subset of the space:

$$
y=L^{2}(0, T ; \mathcal{M}(\mathcal{O})) \times L^{2}((0, T) \times \mathcal{O}) .
$$

Remark 17. We note that the cost functional (63) is well defined on $\Xi_{\varepsilon}$ for every $\varepsilon>0$. Indeed, let $\left(v^{\varepsilon}, \rho^{\varepsilon}\right)$ be any representative of $\Xi_{\varepsilon}$. By supposition (A1), we have that $f_{2}^{\prime}$ : $\mathbb{R} \rightarrow \mathbb{R}$ is a piecewise linear mapping and $\mu^{\varepsilon} \in \mathcal{W}$. Hence $\left(f_{2}^{\prime}\left(\mu^{\varepsilon}\right)\right) \mu_{x}^{\varepsilon}$ is in $L^{2}((0, T) \times \mathcal{O})$, and $v^{\varepsilon} \in L^{2}(0, T ; \mathcal{M}(\mathcal{O}))$ by the definition of the class $\mathcal{V}_{\mathrm{ad}}$. Since $L^{2}((0, T) \times \mathcal{O}) \subset$ $L^{2}(0, T ; \mathcal{M}(\mathcal{O}))$, we come to the required conclusion.

We define the $\tau$-topology on $\mathcal{Y}$ as follows: $\tau$ is the product of the weak-* topology of $L^{2}(0, T ; \mathcal{M}(\mathcal{O}))$ and the topology of norm in $L^{2}((0, T) \times \mathcal{O})$. Then we have the following topological properties of the set $\Xi_{\varepsilon}$ of admissible solutions to the perturbed optimization problem (62)-(63).

Lemma 18. Assume that supposition (A1) holds true. Then the set $\Xi_{\varepsilon}$ is nonempty and sequentially $\tau$-closed for every $\varepsilon>0$.

Proof. For a fixed $\varepsilon>0$ let $\left(u^{\varepsilon}, v^{\varepsilon}\right) \in \mathcal{U}_{\mathrm{ad}} \times \mathcal{V}_{\text {ad }}$ be an arbitrary pair of source terms. Then Theorem 14 implies the existence of a unique pair $\left(\rho^{\varepsilon}, \mu^{\varepsilon}\right)$ such that $\rho^{\varepsilon}=\rho^{\varepsilon}\left(v^{\varepsilon}\right)$ and $\mu^{\varepsilon}=\mu^{\varepsilon}\left(u^{\varepsilon}\right)$ are the corresponding weak solutions to the initial boundary value problem (32)-(34), (38). Since

$$
\begin{aligned}
& \mu^{\varepsilon}, \rho^{\varepsilon} \in \mathcal{W} \\
& :=\left\{y: y \in L^{2}\left(0, T ; H_{0}^{1}(\mathcal{O})\right), \frac{\partial y}{\partial t} \in L^{2}\left(0, T ; H^{-1}(\mathcal{O})\right)\right\}
\end{aligned}
$$

and $\mathcal{W} \hookrightarrow L^{2}((0, T) \times \mathcal{O})$, we conclude that $\left(v^{\varepsilon}, \rho^{\varepsilon}\right) \in \Xi_{\varepsilon}$ and hence $\Xi_{\varepsilon} \neq \varnothing$.

To establish the $\tau$-closedness of $\Xi_{\varepsilon}$, we fix an arbitrary $\tau$ converging sequence of admissible solutions to the perturbed problem (32)-(38) and (62) $\left\{\left(v_{k}^{\varepsilon}, \rho_{k}^{\varepsilon}\right) \in \Xi_{\varepsilon}\right\}_{k=1}^{\infty}$ and show that $\left(v_{*}^{\varepsilon}, \rho_{*}^{\varepsilon}\right) \in \Xi_{\varepsilon}$, where $\left(v_{*}^{\varepsilon}, \rho_{*}^{\varepsilon}\right)$ is its $\tau$-limit.

We have that $v_{k}^{\varepsilon} \stackrel{*}{\rightarrow} v_{*}^{\varepsilon}$ in $L^{2}(0, T ; \mathcal{M}(\mathcal{O}))$ and $\rho_{k}^{\varepsilon} \rightarrow \rho_{*}^{\varepsilon}$ in $L^{2}((0, T) \times \mathcal{O})$. Hence $v_{*}^{\varepsilon} \in \mathcal{V}_{\text {ad }}$ and it remains to show that $\rho_{*}^{\varepsilon}$ is the corresponding weak solution of the initialboundary value problem (32), $(34)_{1}$, and $(38)_{1}$. Indeed, 
in view of the a priori estimate (48), it is easy to see that the $L^{2}\left((0, T) \times \mathcal{O}\right.$-limit function $\rho_{*}^{\varepsilon}$ belongs to the space $\mathcal{W}$ and satisfies conditions:

$$
\begin{array}{r}
f_{1}\left(\rho_{k}^{\varepsilon}\right) \longrightarrow f_{1}\left(\rho_{*}^{\varepsilon}\right), \quad \text { in } L^{2}\left(0, T ; L^{2}(\mathcal{O})\right) \text { as } k \longrightarrow \infty, \\
\left(\rho_{k}^{\varepsilon}\right)_{x} \rightarrow\left(\rho_{*}^{\varepsilon}\right)_{x}, \quad \text { in } L^{2}\left(0, T ; L^{2}(\mathcal{O})\right) \text { as } k \longrightarrow \infty, \\
\left(\rho_{k}^{\varepsilon}\right)_{t} \rightarrow\left(\rho_{*}^{\varepsilon}\right)_{t}, \quad \text { in } L^{2}\left(0, T ; H^{-1}(\mathcal{O})\right) \text { as } k \longrightarrow \infty, \\
\rho_{k}^{\varepsilon}, \rho_{*}^{\varepsilon} \in C\left([0, T] ; L^{2}(\mathcal{O})\right), \quad \rho_{k}^{\varepsilon}(0, x)=\rho_{0}(x) \text { in } \mathcal{O}, \\
\forall k \in \mathbb{N} .
\end{array}
$$

This enables us to pass to the limit in the integral identity (46) as $k \rightarrow \infty$ with $\rho^{\varepsilon}=\rho_{k}^{\varepsilon}$ and $v=v_{k}^{\varepsilon}$, and eo ipso to show that the limit function $\rho_{*}^{\varepsilon}$ is a weak solution to the parabolic problem (32), $(34)_{1},(38)_{1}$.

Thus, the pair $\left(v_{*}^{\varepsilon}, \rho_{*}^{\varepsilon}\right)$ is an admissible solution to the perturbed optimization problem (32)-(38), (62). The proof is complete.

In conclusion of this section, we prove that the penalized problem (32)-(38), (62) has a nonempty set of optimal solutions.

Theorem 19. Assume that supposition (A1) holds true. Then for every $\varepsilon>0$ and $u^{\varepsilon} \in \mathcal{U}_{\text {ad }}$ there exists at least one pair $\left(v_{0}^{\varepsilon}, \rho_{0}^{\varepsilon}\right) \in \Xi_{\varepsilon}$ such that

$$
I_{\varepsilon}\left(v_{0}^{\varepsilon}, \rho_{0}^{\varepsilon}\right)=\inf _{\left(v^{\varepsilon}, \rho^{\varepsilon}\right) \in \Xi_{\varepsilon}} I_{\varepsilon}\left(v^{\varepsilon}, \rho^{\varepsilon}\right)
$$

that is, the problem (32)-(38), (62) is solvable.

Proof. Since $\Xi_{\varepsilon} \neq \varnothing$ and the cost functional $I_{\varepsilon}$ is bounded below on $\Xi_{\varepsilon}$, it follows that there exists a sequence $\left\{\left(v_{k}^{\varepsilon}, \rho_{k}^{\varepsilon}\right)\right\}_{k \in \mathbb{N}} \subset \Xi_{\varepsilon}$ such that

$$
I_{\mathcal{\varepsilon}}\left(v_{k}^{\varepsilon}, \rho_{k}^{\varepsilon}\right) \underset{k \rightarrow \infty}{\longrightarrow} I_{\mathcal{\varepsilon}}^{\min } \equiv \inf _{\left(v^{\varepsilon}, \rho^{\varepsilon}\right) \in \Xi_{\varepsilon}} I_{\varepsilon}\left(v^{\varepsilon}, \rho^{\varepsilon}\right) \geq 0,
$$

that is, $\left\{\left(v_{k}^{\varepsilon}, \rho_{k}^{\varepsilon}\right)\right\}_{k \in \mathbb{N}} \subset \Xi_{\varepsilon}$ is a minimizing sequence for the problem (32)-(38), (62).

To begin with, we show that for any $\lambda>0$ the set

$$
\Xi_{\varepsilon}^{\lambda}=\left\{\left(v^{\varepsilon}, \rho^{\varepsilon}\right) \in \Xi_{\varepsilon}: I_{\mathcal{\varepsilon}}\left(v^{\varepsilon}, \rho^{\varepsilon}\right) \leq \lambda\right\}
$$

is bounded in $L^{2}(0, T ; \mathcal{M}(\mathcal{O})) \times \mathcal{W}$. Indeed, as follows from inequality (68), the sequence of fictitious controls $\left\{v_{k}^{\varepsilon}\right\}_{k \in \mathbb{N}}$ is bounded in $L^{2}(0, T ; \mathcal{M}(\mathcal{O}))$. Hence, we may assume that there exists an element $v_{0}^{\varepsilon} \in \mathcal{V}_{\mathrm{ad}}$ such that $v_{k}^{\varepsilon} \stackrel{*}{\rightarrow} v_{0}^{\varepsilon}$ in $L^{2}(0, T ; \mathcal{M}(\mathcal{O}))$ as $k \rightarrow \infty$, that is,

$$
\begin{aligned}
\lim _{k \rightarrow \infty} \int_{0}^{T}\left\langle v_{k}^{\varepsilon}(t, \cdot), \varphi\right\rangle_{M(\mathcal{O}), C_{0}(\mathcal{O})} \psi(t) d t \\
=\int_{0}^{T}\left\langle v_{0}^{\varepsilon}(t, \cdot), \varphi\right\rangle_{M(\mathcal{O}), C_{0}(\mathcal{O})} \psi(t) d t \\
\quad \forall \varphi \in C_{0}(\mathcal{O}), \quad \forall \psi \in C_{0}^{\infty}(0, T) .
\end{aligned}
$$

Then having used the a priori estimate (48), we see that $\left\{\rho_{k}^{\varepsilon}=\rho^{\varepsilon}\left(v_{k}^{\varepsilon}\right)\right\}_{k \in \mathbb{N}}$ form a uniformly bounded sequence in $\mathcal{W}$. Hence, we may again assume that, up to a subsequence, there exists an element $\rho_{0}^{\varepsilon} \in \mathcal{W}$ such that $\rho_{k}^{\varepsilon} \rightarrow \rho_{0}^{\varepsilon}$ weakly in $\mathcal{W}$ and strongly in $L^{2}((0, T) \times \mathcal{O})$. As a result, $\left(v_{0}^{\varepsilon}, \rho_{0}^{\varepsilon}\right) \in \Xi_{\varepsilon}$ by Lemma 18.

Let us show that the $\tau$-limit pair $\left(v_{0}^{\varepsilon}, \rho_{0}^{\varepsilon}\right)$ is an optimal solution to the penalized problem (32)-(38), (62). Indeed, taking into account supposition (A1) and Theorem 14, we have

$$
\left[\left(f_{2}\left(\mu^{\varepsilon}\right)\right)_{x}-v_{k}^{\varepsilon}\right] \stackrel{*}{\rightarrow}\left(f_{2}\left(\mu^{\varepsilon}\right)\right)_{x}-v_{0}^{\varepsilon}, \quad \text { in } L^{2}(0, T ; \mathcal{M}(\mathcal{O})) .
$$

Using the property of lower semi-continuity for $I_{\mathcal{E}}$ with respect to the $\tau$-topology, we get

$$
0 \leq I_{\mathcal{\varepsilon}}\left(v_{0}^{\varepsilon}, \rho_{0}^{\varepsilon}\right) \leq \lim _{k \rightarrow \infty} I\left(v^{\varepsilon}, \rho^{\varepsilon}\right)=I_{\varepsilon}^{\min } .
$$

Thus the pair $\left(u_{0}^{\varepsilon}, \rho_{0}^{\varepsilon}\right)$ is optimal for the problem (32)-(38), (62).

\section{Approximation Properties of the Perturbed Optimization Problem}

The aim of this section is to study the asymptotic behavior of the optimal solutions to the penalized optimization problem (32)-(38), (62) as the small parameter $\varepsilon$ tends to zero. To begin with, we note that for every $\varepsilon>0$ the set of admissible solutions $\Xi_{\varepsilon}$ is embedded in the topological space $\left(\mathcal{Y}_{1}, \sigma\right)$, where

$$
y_{1}=L^{2}(0, T ; \mathcal{M}(\mathcal{O})) \times L^{2}\left(0, T ; H^{-1}(\mathcal{O})\right),
$$

and $\sigma$ is the product of the weak-* topology of $L^{2}(0, T ; \mathcal{M}(\mathcal{O}))$ and the strong topology of $L^{2}\left(0, T ; H^{-1}(\mathcal{O})\right)$. So, we can take $\sigma$ as the main topology for the asymptotic analysis.

Lemma 20. Let $u^{*}=\sum_{k=1}^{N} u_{k}^{*}(t) \delta_{\tau_{k}} \in U_{\text {ad }}$ a given source term with prescribed location $a<\tau_{1}<\ldots<\tau_{N}<b$. Let $\left\{\mu^{\varepsilon}\right\}_{\varepsilon>0}$ be a sequence of corresponding weak solutions to the initial boundary value problem (33), $(34)_{2},(38)_{2}$ when the small parameter $\varepsilon>0$ varies in a strictly decreasing sequence of positive numbers converging to 0 . Let $\left\{\left(v_{0}^{\varepsilon}, \rho_{0}^{\varepsilon}\right) \in \Xi_{\varepsilon}\right\}_{\varepsilon>0}$ be a sequence of optimal solutions to the penalized problem (32)-(38), (62). Assume that the fictitious controls $\left\{v_{0}^{\varepsilon}\right\}_{\varepsilon>0}$ are bounded in $L^{2}(0, T ; \mathcal{M}(\mathcal{O}))$ and supposition (A1) holds true. Then subsequences of $\left\{\mu^{\varepsilon}\right\}_{\varepsilon>0}$ and of $\left\{\left(v_{0}^{\varepsilon}, \rho_{0}^{\varepsilon}\right)\right\}_{\varepsilon>0}$, still denoted by the suffix $\varepsilon$, can be extracted such that

(a) $v_{0}^{\varepsilon} \stackrel{*}{\rightarrow} v^{*}$ in $L^{2}(0, T ; \mathcal{M}(\mathcal{O}))$;

(b) $\rho_{0}^{\varepsilon} \rightarrow \rho^{*}$ and $\mu^{\varepsilon} \rightarrow \mu^{*}$ weakly in $L^{2}((0, T) \times \mathcal{O})$ and strongly in $L^{2}\left(0, T ; H^{-1}(\mathcal{O})\right)$;

(c) $\left(\rho^{*}, \mu^{*}\right)$ is a weak solution in $\left[L^{2}((0, T) \times \mathcal{O})\right]^{2}$ of the Cauchy problem:

$$
\begin{gathered}
\rho_{t}^{*}+\left(f_{1}\left(\rho^{*}\right)\right)_{x}=v^{*}, \quad \rho^{*}(0, \cdot)=\rho_{0}, \\
\mu_{t}^{*}-\mu_{x}^{*}=u^{*}, \quad \mu^{*}(0, \cdot)=\mu_{0} .
\end{gathered}
$$


Proof. As follows from the a priori estimates (50)-(51), the sequences $\left\{\rho_{0}^{\varepsilon}\right\}_{\varepsilon>0}$ and $\left\{\mu^{\varepsilon}\right\}_{\varepsilon>0}$ are bounded in

$$
\mathcal{W}_{1}=\left\{y: y \in L^{2}\left(0, T ; L^{2}(\mathcal{O})\right), \frac{\partial y}{\partial t} \in L^{2}\left(0, T ; H^{-1}(\mathcal{O})\right)\right\} .
$$

Hence the compactness properties (a)-(b) of the sequences $\left\{v_{0}^{\varepsilon}\right\}_{\varepsilon>0},\left\{\rho_{0}^{\varepsilon}\right\}_{\varepsilon>0}$, and $\left\{\mu^{\varepsilon}\right\}_{\varepsilon>0}$ are a direct consequence of the initial suppositions, the Banach-Alaoglu Theorem, and the compactness embedding $\mathcal{W}_{1} \hookrightarrow L^{2}\left(0, T ; H^{-1}(\mathcal{O})\right)$. Moreover, as follows from estimates (48)-(49), the sequence $\left\{\left(\rho_{0}^{\varepsilon}, \mu^{\varepsilon}\right)\right\}_{\varepsilon>0}$ is bounded in $L^{2}\left(0, T ; L^{2}(\mathcal{O})\right)$. So, we can suppose that

$$
\rho_{0}^{\varepsilon} \longrightarrow \rho^{*}, \quad \mu^{\varepsilon} \longrightarrow \mu^{*}, \quad \text { as } \varepsilon \longrightarrow 0
$$

strongly in $L^{2}\left(0, T ; H^{-1}(\mathcal{O})\right)$ and weakly in $L^{2}\left(0, T ; L^{2}(\mathcal{O})\right)$. In view of estimates (48)-(49), there are elements $\eta, \hat{\eta} \in$ $L^{2}((0, T) \times \mathcal{O})$ such that, up to subsequences, we have

$$
\begin{aligned}
\sqrt{\varepsilon}\left(\rho_{0}^{\varepsilon}\right)_{x} \rightarrow \eta, & \sqrt{\varepsilon}\left(\mu^{\varepsilon}\right)_{x}-\hat{\eta} \quad \text { in } L^{2}((0, T) \times \mathcal{O}), \\
\text { as } \varepsilon & \longrightarrow 0 .
\end{aligned}
$$

In order to verify the item (c), we note that the integral identity (46) leads us to the following relation:

$$
\begin{gathered}
\int_{0}^{T} \int_{\mathcal{O}}\left[-\rho_{0}^{\varepsilon} \frac{\partial \varphi}{\partial t}+\sqrt{\varepsilon}\left(\sqrt{\varepsilon} \frac{\partial \rho_{0}^{\varepsilon}}{\partial x}\right) \frac{\partial \varphi}{\partial x}-f_{1}\left(\rho_{0}^{\varepsilon}\right) \frac{\partial \varphi}{\partial x}\right] d x d t \\
=\int_{0}^{T}\left\langle v_{0}^{\varepsilon}(t, \cdot), \varphi(t, \cdot)\right\rangle_{\mathcal{M}(\mathcal{O}), C_{0}(\mathcal{O})} d t,
\end{gathered}
$$

which holds true for every $\varepsilon>0$ and any test function $\varphi \in$ $C_{0}^{\infty}((0, T) \times \mathcal{O})$. Since $v_{0}^{\varepsilon} \stackrel{*}{\rightarrow} v^{*}$ in $L^{2}(0, T ; \mathcal{M}(\mathcal{O}))$ as $\mathcal{\varepsilon} \rightarrow 0$, we can pass to the limit in (79) using the property $(77)_{1}$ $(78)_{1}$. As a result, we come to the relation

$$
\begin{aligned}
\int_{0}^{T} \int_{\mathcal{O}} & {\left[-\rho^{*} \frac{\partial \varphi}{\partial t}-f_{1}\left(\rho^{*}\right) \frac{\partial \varphi}{\partial x}\right] d x d t } \\
= & \int_{0}^{T}\left\langle v^{*}(t, \cdot), \varphi(t, \cdot)\right\rangle_{\mathcal{M}(\mathcal{O}), C_{0}(\mathcal{O})} d t,
\end{aligned}
$$

which gives us the weak formulation of the hyperbolic conservation law $(75)_{1}$. As for the initial condition $(75)_{1}$, we note that by continuity property (41) the following identity

$$
\lim _{t \rightarrow 0+} \frac{1}{t} \int_{0}^{t}\left|\int_{\mathcal{O}}\left(\rho_{0}^{\varepsilon}(s, \cdot)-\rho_{0}\right) \psi d x\right| d s=0, \quad \forall \psi \in C_{0}^{\infty}(\mathcal{O})
$$

is valid for every $\varepsilon>0$. So, we can pass to the limit in $(81)$ as $\varepsilon \rightarrow 0$ using the weak convergence of $\rho_{0}^{\varepsilon} \rightarrow \rho^{*}$ in $L^{2}((0, T) \times$ $\mathcal{O})$. As a result, the initial condition for the limit function $\rho^{*}$ is satisfied in the following sense:

$$
\lim _{t \rightarrow 0+} \frac{1}{t} \int_{0}^{t}\left|\int_{\mathcal{O}}\left(\rho^{*}(s, \cdot)-\rho_{0}\right) \psi d x\right| d s=0, \quad \forall \psi \in C_{0}^{\infty}(\mathcal{O}) .
$$

Thus, $\rho^{*} \in L^{2}((0, T) \times \mathcal{O})$ is a weak solution to the Cauchy problem (74). By analogy, similar properties for the limit function $\mu^{*}$ can be proved. This concludes the proof.

The next result is crucial in this paper. We show that approximately entropy weak solutions to the system of nonlinear conservation laws with impulse controls can be constructed by optimal solutions to the penalized problem (32) $-(38)$ and (62).

Theorem 21. Let $u=\sum_{k=1}^{N} u_{k}(t) \delta_{\tau_{k}} \in u_{a d}$ be a given source term with prescribed location $a<\tau_{1}<\cdots<\tau_{N}<b$. Assume that there exists a sequence of pairs $\left\{\left(\hat{v}^{\varepsilon}, \hat{\rho}^{\varepsilon}\right) \in \Xi_{\varepsilon}\right\}_{\varepsilon>0}$ satisfying the following relation:

$$
\limsup _{\varepsilon \rightarrow 0} I_{\varepsilon}\left(\hat{v}^{\varepsilon}, \hat{\rho}^{\varepsilon}\right)<+\infty .
$$

Let $\left\{\left(v_{0}^{\varepsilon}, \rho_{0}^{\varepsilon}\right) \in \Xi_{\varepsilon}\right\}_{\varepsilon>0}$ be a sequence of optimal solutions to the penalized problem (32)-(38) and (62). Then, under supposition (A1), for every $\sigma$-cluster point $\left(v^{*}, \rho^{*}\right) \in \mathcal{Y}_{1}$ of the sequence $\left\{\left(v_{0}^{\varepsilon}, \rho_{0}^{\varepsilon}\right) \in \Xi_{\varepsilon}\right\}_{\varepsilon>0}$ we has that the triplet $\left(u^{*}, \rho^{*}, \mu^{*}\right)$ is an approximately entropy solution to the Cauchy problem (14)-(16) in the domain $(0, T) \times \mathcal{O}$ and the equality $v^{*}=$ $\left(f_{2}\left(\mu^{*}\right)\right)_{x}$ is valid almost everywhere in $(0, T) \times \mathcal{O}$. Here the distribution $\mu^{*}$ is defined by (75).

Remark 22. It is worth to notice that the existence of a sequence $\left\{\left(\hat{v}^{\varepsilon}, \hat{\rho}^{\varepsilon}\right) \in \Xi_{\varepsilon}\right\}_{\varepsilon>0}$ satisfying relation (83) is rather important for our further analysis and this assumption is coming from the regularity property of the original Cauchy problem (14)-(18). Here by the regularity of Cauchy problem (14)-(18) we mean that this problem admits at least one entropy solution. Since the existence of such solutions is unknown in general, we must assume it. Only in this case it has a sense to construct an approximation of entropy solutions. So, for the regular Cauchy problem (14)-(18), the sequence $\left\{\left(\hat{v}^{\varepsilon}, \hat{\rho}^{\varepsilon}\right) \in \Xi_{\varepsilon}\right\}_{\varepsilon>0}$ can be constructed as follows: $\hat{v}^{\varepsilon}=\left(f_{2}\left(\mu^{\varepsilon}\right)\right)_{x}$ for all $e>0$, and $\left(\rho_{\varepsilon}, \mu_{\varepsilon}\right)$ is the corresponding solution of the perturbed problem (32)-(37). As for the general case, we demand the fulfilment of the condition (83).

Proof. As Lemma 20 indicates, the sequence $\left\{\left(\mu^{\varepsilon}\right) \in \mathcal{W}_{1}\right\}_{\varepsilon>0}$ is relatively compact with respect to the strong convergence in $L^{2}\left(0, T ; H^{-1}(\mathcal{O})\right)$ and the weak convergence in $L^{2}\left(0, T ; L^{2}(\mathcal{O})\right)$. So, passing to a subsequence, when the occasion requires, we get

$$
\begin{aligned}
\mu^{\varepsilon} \longrightarrow \mu^{*}, & \text { in } L^{2}\left(0, T ; H^{-1}(\mathcal{O})\right), \\
\mu^{\varepsilon} \rightarrow \mu^{*}, & \text { in } L^{2}\left(0, T ; L^{2}(\mathcal{O})\right),
\end{aligned}
$$

where $\mu^{*} \in L^{2}((0, T) \times \mathcal{O})$ is a weak solution to the Cauchy problem (75). For our further analysis we have to show that

$$
\left(f_{2}\left(\mu^{\varepsilon}\right)\right)_{x} \rightarrow\left(f_{2}\left(\mu^{*}\right)\right)_{x}, \quad \text { in } L^{2}\left(0, T ; H^{-1}(\mathcal{O})\right) .
$$


Indeed, let $\varphi \in C_{0}^{\infty}((0, T) \times \mathcal{O})$ be a fixed test function. Then the following estimate holds:

$$
\begin{aligned}
& \left|\int_{0}^{T} \int_{\mathcal{O}} \mu_{x}^{\varepsilon} \varphi d x d t\right| \\
& =\left|\int_{0}^{T} \int_{\mathcal{O}}\left(\mu_{t}^{\varepsilon} \varphi+\mu_{x}^{\varepsilon} \varphi_{x}-u \varphi\right) d x d t\right| \\
& \leq \int_{0}^{T}\left(\left|\left\langle\mu_{t}^{\varepsilon}, \varphi\right\rangle_{H^{-1}(\mathcal{O}), H_{0}^{1}(\mathcal{O})}\right|+\sqrt{\varepsilon} \int_{\mathcal{O}}\left|\sqrt{\varepsilon} \mu_{x}^{\varepsilon} \varphi_{x}\right| d x\right. \\
& \left.+\left|\langle u, \varphi\rangle_{H^{-1}(\mathcal{O}), H_{0}^{1}(\mathcal{O})}\right|\right) d t \\
& \stackrel{\text { by (59),(61) }}{\leq}\left(C+\|u\|_{L^{2}\left(0, T ; H^{-1}(\mathcal{O})\right)}\right)\|\varphi\|_{L^{2}\left(0, T ; H_{0}^{1}(\mathcal{O})\right)} \text {. }
\end{aligned}
$$

Hence the sequence $\left\{\mu_{x}^{\varepsilon}\right\}_{\varepsilon>0}$ is uniformly bounded in $L^{2}(0, T$; $H^{-1}(\mathcal{O})$. Therefore, in view of $(84)$, we can suppose that $\mu_{x}^{*} \in L^{2}\left(0, T ; H^{-1}(\mathcal{O})\right)$ and

$$
\mu_{x}^{\varepsilon} \stackrel{*}{\rightarrow} \mu_{x}^{*}, \quad \text { in } L^{2}\left(0, T ; H^{-1}(\mathcal{O})\right) .
$$

As a result, applying the arguments of Remark 17, we come to the required conclusion (85).

Let $\left\{\left(\hat{v}^{\varepsilon}, \hat{\rho}^{\varepsilon}\right) \in \Xi_{\varepsilon}\right\}_{\varepsilon>0}$ be a sequence with property (83). Then there exist a value $\varepsilon_{0}>0$ and a constant $c>0$ independent of $\varepsilon$ such that the following inequality holds true:

$$
\begin{aligned}
& \left\|v_{0}^{\varepsilon}\right\|_{L^{2}(0, T ; \mathcal{M}(\mathcal{O}))}^{2}+\varepsilon^{-1}\left\|\left(f_{2}\left(\mu^{\varepsilon}\right)\right)_{x}-v_{0}^{\varepsilon}\right\|_{L^{2}\left(0, T ; H^{-1}(\mathcal{O})\right)} \\
& \quad \leq I_{\mathcal{\varepsilon}}\left(\widehat{v}^{\varepsilon}, \hat{\rho}^{\varepsilon}\right) \leq \mathcal{c}, \quad \forall \mathcal{\varepsilon} \in\left(0, \varepsilon_{0}\right) .
\end{aligned}
$$

Hence the sequence of optimal fictitious controls $\left\{v_{0}^{\varepsilon}\right\}_{\varepsilon>0}$ is bounded in $L^{2}(0, T ; \mathcal{M}(\mathcal{O}))$. Therefore, by Lemma 20 the sequence of optimal pairs $\left\{\left(v_{0}^{\varepsilon}, \rho_{0}^{\varepsilon}\right) \in \Xi_{\mathcal{\varepsilon}}\right\}_{\mathcal{\varepsilon}>0}$ is relatively compact with respect to the $\sigma$-topology of $L^{2}(0, T ; \mathcal{M}(\mathcal{O})) \times$ $L^{2}\left(0, T ; H^{-1}(\mathcal{O})\right)$. Moreover, every $\sigma$-cluster point $\left(v^{*}, \rho^{*}\right) \in$ $\mathcal{y}_{1}$ possesses the properties (a)-(c) of Lemma 20.

Further we note that the inequality (88) leads to the estimate

$$
0 \leq\left\|\left(f_{2}\left(\mu^{\varepsilon}\right)\right)_{x}-v_{0}^{\varepsilon}\right\|_{L^{2}\left(0, T ; H^{-1}(\mathcal{O})\right)} \leq \mathcal{E} \mathcal{C}, \quad \forall \mathcal{\varepsilon} \in\left(0, \varepsilon_{0}\right) .
$$

Since $\left(f_{2}\left(\mu^{\varepsilon}\right)\right)_{x}-v_{0}^{\varepsilon} \rightarrow\left(f_{2}\left(\mu^{*}\right)\right)_{x}-v^{*}$ in $L^{2}\left(0, T ; H^{-1}(\mathcal{O})\right)$ (see $(85)), v_{0}^{\varepsilon} \stackrel{*}{\rightarrow} v^{*}$ in $L^{2}(0, T ; \mathcal{M}(\mathcal{O}))$, and $\mathcal{M}(\mathcal{O}) \subset$ $H^{-1}(\mathcal{O})$, we can pass to the limit in $(89)$ as $\varepsilon \rightarrow 0$. Then, in view of the lower semicontinuity property, we obtain

$$
\begin{aligned}
0 & \leq\left\|\left(f_{2}\left(\mu^{*}\right)\right)_{x}-v^{*}\right\|_{L^{2}\left(0, T ; H^{-1}(\mathcal{O})\right)} \\
& \leq \liminf _{\varepsilon \rightarrow 0}\left\|\left(f_{2}\left(\mu^{\varepsilon}\right)\right)_{x}-v_{0}^{\varepsilon}\right\|_{L^{2}\left(0, T ; H^{-1}(\mathcal{O})\right)} \leq 0 .
\end{aligned}
$$

Since this is equivalent to the equality $v^{*}=\left(f_{2}\left(\mu^{*}\right)\right)_{x}$ almost every where in $(0, T) \times \Omega$, by Lemma 15 it follows that the pair $\left(\rho^{*}, \mu^{*}\right)$ is an approximately entropy solution to the initial-boundary value problem (32)-(38). This concludes the proof.

\section{Conclusion}

In this article, we have proposed the approximation of entropy solutions for the system of two hyperbolic conservation laws (14)-(16) with impulse source terms. We have considered the case when influx-rates in the second equation (15) take the form of impulse functions (17)-(18). Since the existence of entropy solutions for Cauchy problem (14)(18) is not covered by the classical theory, we combine the vanishing viscosity method and the so-called principle of fictitious controls in order to show that entropy solutions to the original Cauchy problem can be approximated by optimal solutions of special optimization problems. The main result is given by Theorem 21, where we conclude that every $\sigma$ cluster pair $\left(v^{*}, \rho^{*}\right) \in \mathcal{Y}_{1}$ of the sequence $\left\{\left(v_{0}^{\varepsilon}, \rho_{0}^{\varepsilon}\right) \in \Xi_{\varepsilon}\right\}_{\varepsilon>0}$ of optimal solutions to the penalized problem (32)-(38), (62) is an approximately entropy solution $\left(u^{*}, \rho^{*}, \mu^{*}\right)$ to the Cauchy problem (14)-(16).

\section{References}

[1] A. Bressan, Hyperbolic Systems of Conservation Laws-The One-dimensional Cauchy Problem, Oxford University Press, Oxford, UK, 2000.

[2] S. Kruzhkov, "First-order quasilinear equations in several independent variables," Mathematics of the USSR-Sbornik, vol. 10, pp. 217-243, 1970.

[3] P. D. Lax, Hyperbolic System of Conservation Laws and the Mathematical Theory of Shock Waves, SIAM, Philadelphia, Pa, USA, 1973.

[4] C. D'apice and R. Manzo, "A fluid dynamic model for supply chains," Networks and Heterogeneous Media, vol. 1, no. 3, pp. 379-398, 2006.

[5] E. Giusti, Minimal Surfaces and Functions of Bounded Variation, Birkhäuser, Boston, Mass, USA, 1984.

[6] M. Garavello and B. Piccoli, Traffic Flow on Networks, vol. 1 of AIMS Series on Applied Mathematics, 2006.

[7] G. A. Chechkin and A. Yu. Goritsky, "S. N. Kruzhkov's lectures on first-order quasilinear PDEs," Analytical and Numerical Aspects of PDEs, pp. 1-67, 2010.

[8] S. Ulbrich, Optimal Control of Nonlinear Hyperbolic Conservation Laws with Source Terms, Fakultät für Mathematik,Technische Universität München, 2002.

[9] D. Armbruster, D. Marthaler, and C. Ringhofer, "Kinetic and fluid model hierarchies for supply chains," SIAM Journal on Multiscale Modeling and Simulation, vol. 2, pp. 43-61, 2004.

[10] S. Göttlich, M. Herty, and A. Klar, "Modelling and optimization of supply chains on complex networks," Communications in Mathematical Sciences, vol. 4, no. 2, pp. 315-330, 2006.

[11] C. Kirchner, M. Herty, S. Göttlich, and A. Klar, "Optimal control for continuous supply network models," Networks and Heterogeneous Media, vol. 1, no. 4, pp. 675-688, 2006.

[12] J. L. Lions, Some Methods of Solving Non-Linear Boundary Value Problems, Dunod-Gauthier-Villars, Paris, France, 1969.

[13] V. A. Solonnikov, "A priori estimates for equations of second order parabolic type," Trudy Matematicheskogo Instituta imeni V.A. Steklova, vol. 70, pp. 133-212, 1960. 

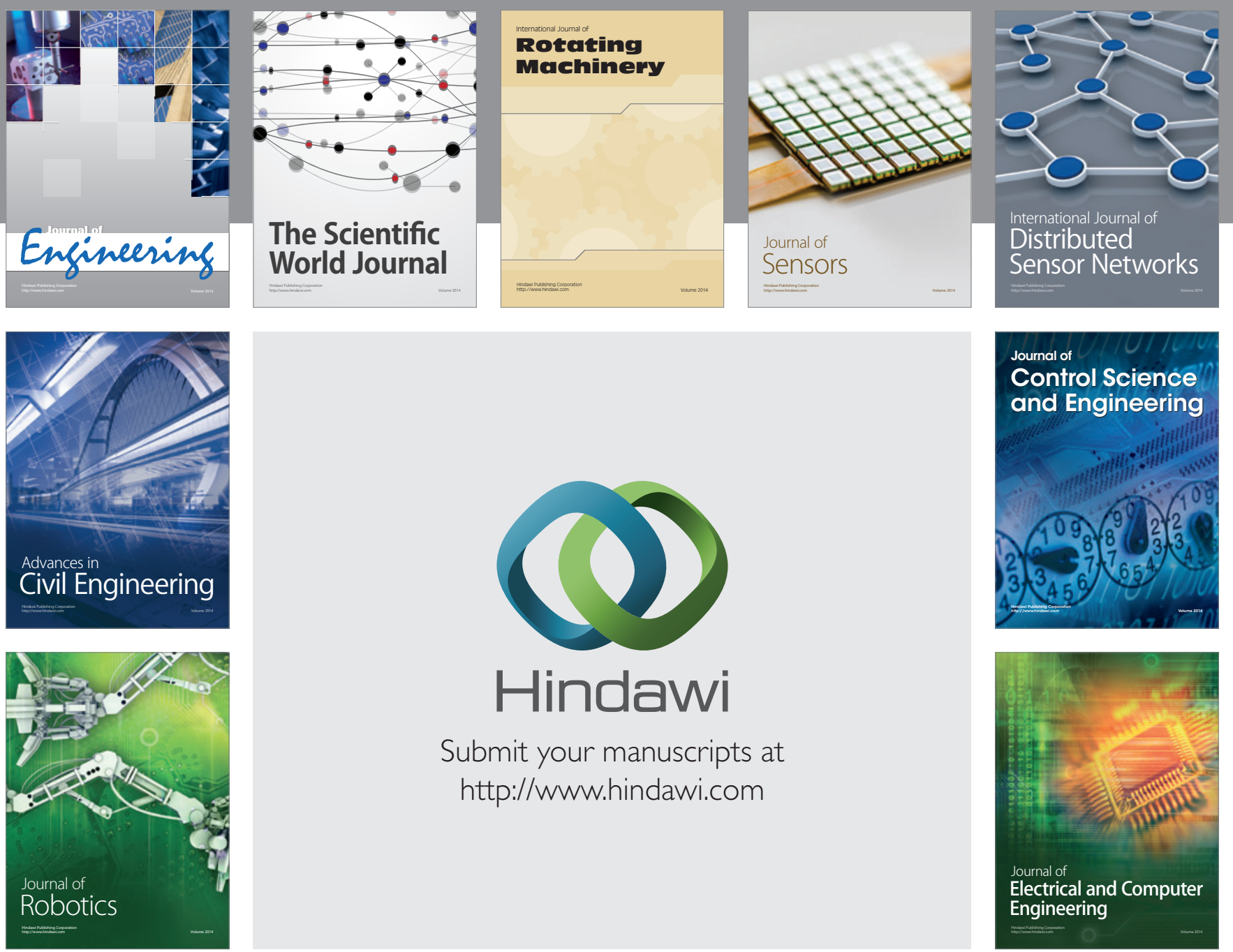

Submit your manuscripts at

http://www.hindawi.com
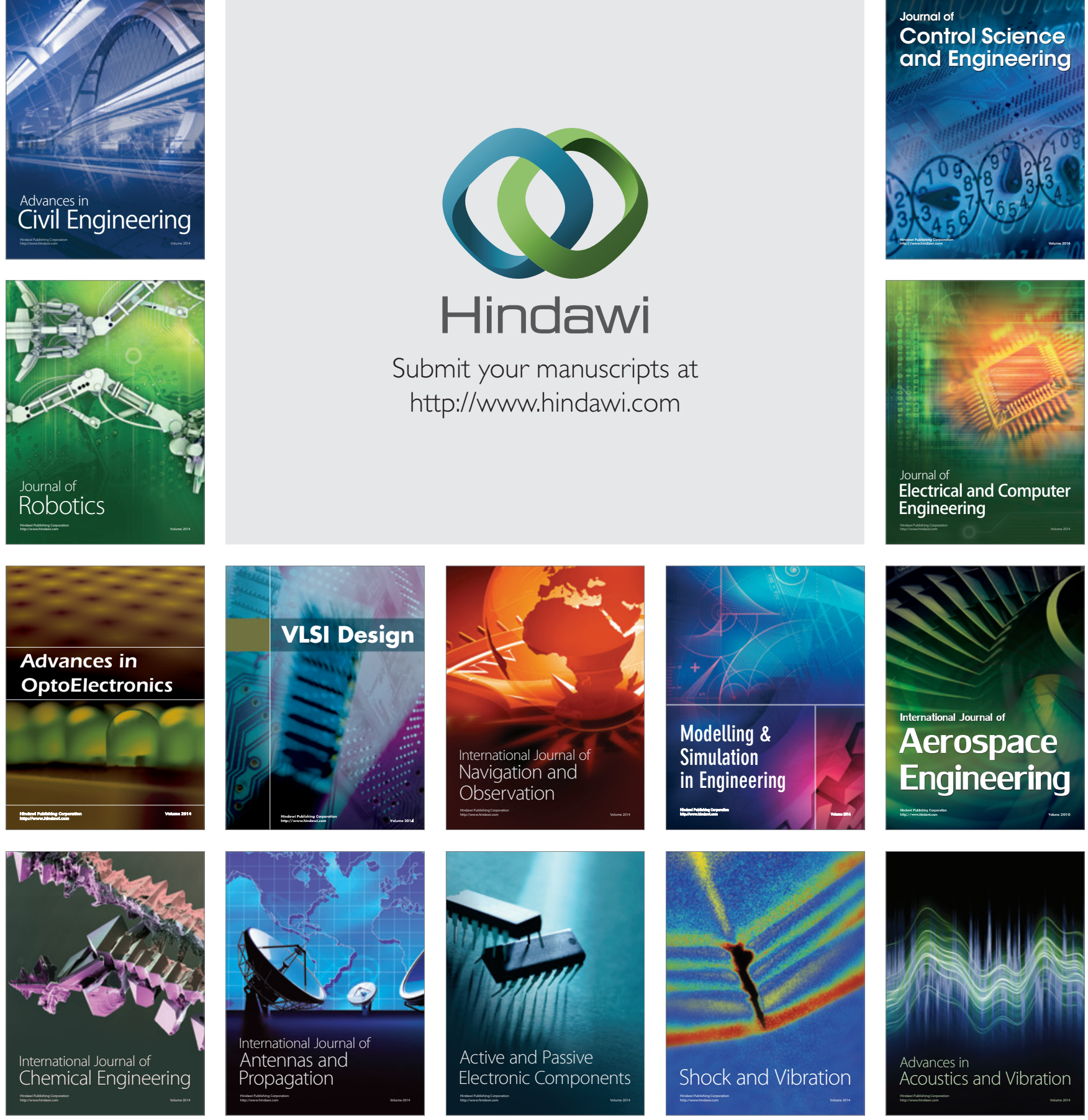